\title{
miR-200a contributes to the migration of BMSCs induced by the secretions of $E$. faecalis via FOXJ1/NFKB/MMPs axis
}

Mingwei $\mathrm{Li}^{1,2,3+}$, Lifan Wei ${ }^{1,2,3 \dagger}$, Wei Zhou ${ }^{2,3}$, Zhiyan $\mathrm{He}^{2,3}$, Shujun Ran ${ }^{1,2,3}$ and Jingping Liang ${ }^{1,2,3^{*}}$

\begin{abstract}
Background: Upon migrating to the injured sites, bone marrow mesenchymal stem cells (BMSCs) play critical roles in the repair of bone lesion caused by chronic apical periodontitis. Emerging evidences have shown that Enterococcus faecalis is always associated with apical periodontitis, especially refractory apical periodontitis. But the mechanism underlying how Enterococcus faecalis affects the migration of BMSCs remains unclear.

Methods: The effects of Enterococcus faecalis supernatants on the migration of BMSCs were determined by transwell migration assays. miRNA sequencing was performed to detect the significantly differentially expressed miRNAs of BMSCs. Proteomics analysis was used to detect the protein expression alterations of BMSCs. Luciferase report assays were deployed to verify the targets of miRNA. Western blot analysis was performed to examine the expressions of matrix metalloproteinases-3, matrix metalloproteinases-9, Forkhead Box Protein J1 (FOXJ1), and nuclear factor kappa B (NFKB). The activations of NFKB were detected by luciferase assays with NFkBluc reporter.
\end{abstract}

Results: We found that Enterococcus faecalis supernatants could promote the migration of BMSCs. The upregulation of miR-200a-3p in this process contributed to BMSC migration through downregulating its target Forkhead Box Protein J1. Moreover, FOXJ1/ NFkB axis was found to regulate matrix metalloproteinases (MMPs) in this process.

Conclusions: These results above suggest that miR-200a contributes to the migration of BMSCs induced by the secretions of $E$. faecalis via FOXJ1/NFKB/MMPs axis.

Keywords: Bone marrow mesenchymal stem cells, Cell migration, Nuclear factor kappa B, Enterococcus faecalis, miRNA, Host-pathogen interactions

\section{Background}

Apical periodontitis, which is characterized by the inflammation and destruction of the apical periodontium, is always caused by the host immune response to microbial infection in the root canal system [1]. Those species originated from intestinal flora, such as E. faecalis, have

\footnotetext{
* Correspondence: liangjp9h@gmail.com

${ }^{\dagger}$ Mingwei Li and Lifan Wei contributed equally to this work.

'Department of Endodontics and Operative Dentistry, Shanghai Ninth People's Hospital, College of Stomatology, Shanghai Jiao Tong University

School of Medicine, Shanghai, China

${ }^{2}$ National Clinical Research Center for Oral Diseases, Shanghai, China

Full list of author information is available at the end of the article
}

been identified as dominant factors causing apical periodontitis [2]. E. faecalis is always isolated as a monoculture in retreated root canals [3]. It belongs to facultative aerobic species and is usually found in secondary infection or post-treatment of apical periodontitis, especially in the refractory inflammation [4]. E. faecalis is tolerated to antimicrobials and contains the ability of surviving in a nutrient-deficient environment. Thus, persisting infections in root canal or apical periodontium are always associated with $E$. faecalis [5]. There are various virulence factors produced by $E$. faecalis, such as lipoteichoic acid, aggregation substance protein, and surface adhesion $[6$, 
7]. During the development of pulpitis, E. faecalis can invade and colonize at dentinal tubules. Studies on the etiology of refractory apical periodontitis have revealed that E. faecalis biofilms in the dentinal tubules contribute to the retaining of apical periodontitis [8].

Since apical periodontitis is characterized by inflammation and bone resorption [9], cells associated with this process should be carefully taken into consideration. Belonging to multipotent stem cells and widely presenting in bone marrow, bone marrow mesenchymal stem cells (BMSCs) can differentiate into osteoblasts, chondrocytes, or adipocytes [10]. Meanwhile, BMSCs have shown certain ability of moving from niche to the peripheral circulation, and further to the target tissues [11]. The recruitment of BMSCs is required for the repair of bone lesion, and the migration of BMSCs is usually attracted by the environmental factors at the site of injury [12]. There are various factors gathering at the injury, including infectious factors and those produced by injured tissues.

With bacterial infection, BMSCs contact with bacterial components and recognize them through the receptors on the cell membrane. Studies on human BMSCs have revealed that lipopolysaccharide (LPS), the cell wall component from gram-positive bacteria, can increase their migration [13], while the synthetic lipopeptide could inhibit the migration of mouse BMSCs [14]. The migration of human dental pulp stem cells were also increased with the stimulation of Toll-like receptor 2 (TLR2) ligands [15]. Studies above remind us that it depends on the type of mesenchymal stem cell in which migration effect would be caused by bacterial components. In fact, it is hard to ensure the regeneration of the tissue without the efficient migration of BMSCs into the injured sites. It requires more acknowledgments in this field to induce BMSCs migrating and generating new tissues.

In recent years, it has been widely accepted that miRNAs play important roles in the biological regulation of stem cells. miRNAs are highly conserved endogenous non-coding RNAs with a length of 19 to 25 nucleotides. They usually act as a negative regulator by binding to the 3'UTR sites of their target mRNAs [16] and further modulate the cell signaling transduction [17]. They also take part in various biological processes, including cell apoptosis, metabolism, migration, and differentiation [18]. Specific miRNAs have been taken as biomarkers and therapeutic targets for their roles in pathological processes and human diseases [19]. A growing number of miRNAs have been explored for their roles in the migration of BMSCs as either inhibitors or activators. Abnormal miRNA expression would also lead to an obvious alteration on the osteogenic differentiation of BMSCs [20]. A previous study has shown that miR-335 overexpression would downregulate the proliferation, migration, and differentiation of human BMSCs [21]. By upregulating the expressions of MMP-2 and MMP-9, miR-21 can promote the migration of BMSCs via the PI3K/Akt pathway [22]. The migration of rat BMSCs could be inhibited by miR-375 via Akt signaling [23].

The objective of this article is to detect miRNAs related to the migration of BMSCs which is induced by $E$. faecalis, the main infection in refractory apical periodontitis. In this study, we investigate the variations of miRNA signaling of BMSCs in response to E. faecalis supernatants (EfS) and evaluate how the miRNAs participate in cell migration. We examine and validate a series of miRNAs which are differentially expressed with the stimulation of $E$. faecalis supernatants, and further elucidate that adjustments of the miRNA can regulate the migration of BMSCs. We provide an insight into the mechanism that miR-200a-3p is involved in the NFKB signaling and affects the expressions of MMPs in the migration of BMSCs. Together, the outcomes of this study provide a better understanding on the movement of BMSCs with the invasion of E. faecalis in apical periodontitis and suggest a novel way to drive BMSCs to the injured sites and complete tissue regeneration.

\section{Methods}

\section{Cell culture}

To obtain BMSCs, femur bones from rats were dissected, isolated, and flushed with PBS. Bone marrow was aspirated and suspended in PBS with 5\% FBS (Gibco). After centrifuged at $400 \mathrm{~g}$ for $5 \mathrm{~min}$ and washed twice with PBS, isolations were cultured in DMEM supplemented with $10 \% \mathrm{FBS}, 100 \mu \mathrm{g} / \mathrm{ml}$ each of penicillin and streptomycin under the condition of $37^{\circ} \mathrm{C}$ and $5 \% \mathrm{CO}_{2}$. Enterococcus faecalis ATCC33186 was cultured in brain heart infusion (BHI) medium and the growth rate was measured by the optical density at $600 \mathrm{~nm}$.

\section{Proteomics analysis}

After treated with mediums containing EfS or BHI for $48 \mathrm{~h}$, cells were collected and analyzed as previously described [24]. In brief, label-free peptide MS1 intensity-based methods were used to identify the levels of proteins in different groups, and LC-MS/MS analysis was performed on a Q Exactive mass spectrometer (Thermo Scientific). Those proteins with level change $>2$ and $p<0.05$ were taken as upregulated in EfS- vs. BHI-BMSCs. While proteins with level change $<0.5$ and $p<0.05$ were taken as downregulated. Blast2Go (https://www.blast2go.com) were used for the functional annotation of proteins. KEGG (http://www. kegg.jp) was employed to conduct pathway enrichment. Fisher's exact test was used in GO and KEGG analysis.

\section{MicroRNA-sequencing}

Cells were treated with indicated mediums for $24 \mathrm{~h}$ and miRNA sequencing was performed as previously described 
[24]. In brief, total RNA was obtained with TRIzol reagent, and then small RNA sequencing libraries were conducted. The libraries were quantified with an Agilent 2100 Bioanalyzer. The raw lllumina sequence data were prepared and converted to fastq files.

\section{Transwell migration assays}

The two-chamber transwell system with $8 \mu \mathrm{m}$ pore size was used in these assays. After treated with indicated medium for $24-48 \mathrm{~h}$, cells were seeded into the upper chamber of the inserts with serum-free medium, while the medium with 10\% FBS was filled into the lower chamber. After incubation for $12 \mathrm{~h}$, the inserts were fixed by $100 \%$ methanol and subsequently stained with $0.1 \%$ crystal violet. The migrated cells on the lower side of the inserts were imaged and counted.

\section{Scratch wound assays}

After obtaining a confluent monolayer, cells were incubated in the serum-free medium for $12 \mathrm{~h}$ and then physically wounded with a sterile pipette tip. Before adding culture medium with BHI or EfS, detached cells were washed away with PBS. The scratches were recorded with a microscope at the defined positions after 0 and $48 \mathrm{~h}$. The scratch wound closures, which were expressed as a percentage of scratch surface area covered by migrated cells, were analyzed with ImageJ.

\section{Western blot analysis}

Bone marrow mesenchymal stem cells between passage 3 and passage 5 were seeded in plates and reached $70-80 \%$ at $37^{\circ} \mathrm{C}$ in $5 \% \mathrm{CO}_{2}$ incubator. Stimulation was applied as the indicated time. And then cells were washed with PBS and lysed in RIPA with protease inhibitors. Proteins were extracted, separated by SDS-PAGE, and transferred to PVDF membranes. Before probing with the secondary antibodies conjugated with HRP for $1 \mathrm{~h}$ at room temperature, the membranes were blocked with $5 \%$ non-fat milk and rinsed in the indicated primary antibodies at $4{ }^{\circ} \mathrm{C}$ overnight. Primary antibodies against MMP3(\#14351, CST), MMP13(\#69926, CST), $\mathrm{NF \kappa B}(\# 8242, \mathrm{CST}), \mathrm{p}-\mathrm{NFkB}(\# 3033, \mathrm{CST}), \beta$-actin (\#4970, CST), and Foxj1 (\#ab235445, Abcam) were applied in this study according to the manufacturers' instructions. Signals were captured using the enhanced chemiluminescence kit and ChemiDoc MP System. $\beta$-actin was shown as the internal control.

\section{Real-time PCR analysis}

After treated with indicated culture medium, cells were lysed and the total RNA extract kit was used to obtain the RNA according to the manufacture's protocol. After converted into cDNA, gene expressions were detected on a light480 real-time PCR system.

\section{Oligonucleotide transfection}

Synthetic miRNA mimics, inhibitors, and negative control oligonucleotides were designed and produced by Ribobio. BMSCs were transfected with miRNA using DharmaFECT Transfection Reagents according to manufacturer's protocols. Briefly, transfections were conducted when cells reached $50-60 \%$ confluence. Total RNA and proteins were obtained after 24 and $48 \mathrm{~h}$, respectively.

\section{Luciferase assays}

After transfected with appropriate plasmids for $48 \mathrm{~h}$, cells were lysed for luciferase assays. Dual-luciferase reporter assay system was used for detection according to manufacturer's protocol and Renilla luciferase activities were taken as an internal control.

\section{Statistical analysis}

All statistical analyses were performed by Graphpad Prism 8.0. Statistical results expressed in the figures are shown as the mean \pm standard deviation calculated from at least three independent experiments. The statistical significance of the differences was analyzed by unpaired Student's $t$ test at a significance level of $p<0.05$.

\section{Results}

\section{E. faecalis supernatants (EfS) in late stationary phase promote the migration of BMSCs}

To address the unsolved questions surrounding how $E$. faecalis affects the role of BMSCs during the repair of periapical bone loss, we obtained the cell-free supernatants of E. faecalis ATCC33186, which was cultured in brain heart infusion (BHI) medium. Following cultured in medium for $15 \mathrm{~h}$, the growth of E. faecalis was recorded according to the optical density at $600 \mathrm{~nm}$ and culture supernatants were harvested in late stationary phase (Fig. 1a). To detect the effect of supernatants on the migration of BMSCs, we performed the transwell assays and scratch wound assays. Compared with the control groups added with BHI medium, the supernatants added into the culture medium dramatically promoted the migration of BMSCs to the lower side of the inserts in transwell (Fig. 1b) and increased the proportion of wound closure area after scratch (Fig. 1c).

\section{miR-200a-3p participates in the regulation of BMSCs migration}

To further address the question of how $E$. faecalis supernatants impact the migration of BMSCs and whether any miRNAs play roles during this regulation, we performed miRNA sequencing to detect the significantly differentially expressed miRNAs. Thirty-eight miRNAs were defined by setting $p$ adj $<0.05$ as thresholds and 10 miRNAs were upregulated in BMSCs with E. faecalis supernatant treatment (Fig. 2a). Considering that miR- 

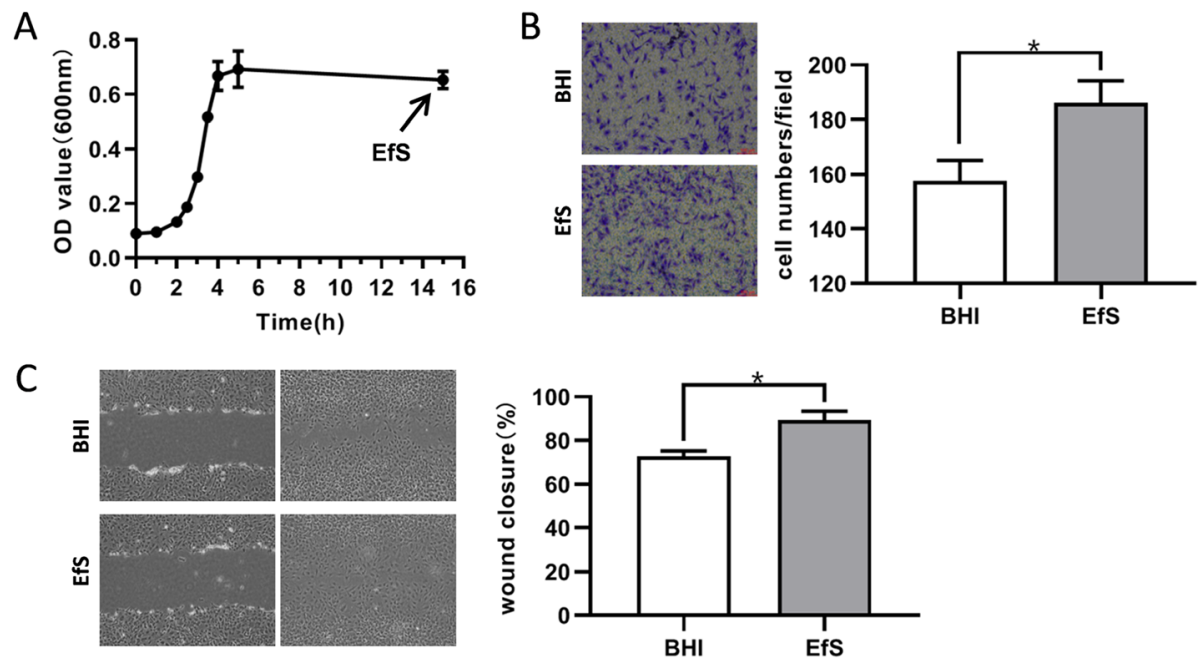

Fig. 1 Enterococcus faecalis supernatants (EfS) increased the migration of rat bone marrow mesenchymal stem cells (BMSCS). a Harvesting of Enterococcus faecalis ATCC33186 cell-free culture supernatants. Enterococcus faecalis ATCC33186 was cultured in brain heart infusion (BHI) medium and culture supernatants were harvested at late stationary phase. The growth was recorded with the optical density of medium over time. $\mathbf{b}$ Observation of BMSCs migration with the stimulation of Enterococcus faecalis supernatants (EfS) in transwell assays. BMSCs were cultured on the upper inserts of transwell in DMEM with EfS. Those cells migrated to the lower side of transwell membrane were fixed, stained with crystal violet, and counted. c Observation of BMSCs migration with the stimulation of EfS in scratch wound assays. The quantitative results are the means \pm SD of three independent experiments. ${ }^{*} p<0.05$

200 family has been proved to take part in the regulation of cell migration, we examined the expressions of miR200a-3p, miR-200b-3p, and miR-429 by qRT-PCR. The results showed that the expressions of miR-200a-3p and miR-200b-3p were dramatically upregulated, while no significant results were obtained with miR-429 (Fig. 2b). The expressions of miR-200a-3p were also identified after transfection of miR-200a-3p mimics or inhibitors (Fig. 2c, d). To investigate the miR-200a-3p function in BMSCs migration, cells were transfected with miR-200a$3 \mathrm{p}$ mimics, inhibitors, or negative control, and transwell assays showed that miR-200a-3p mimics could promote the migration of BMSCs, which could be attenuated by inhibitors (Fig. 2e). With the stimulation of E. faecalis supernatants, the migration of BMSCs transfected with miR-200a-3p mimics could still be decreased by miR200a-3p inhibitors, while no significant result was obtained in comparison with the control group (Fig. 2e).

\section{Proteomic analysis on the BMSCs treated with $E$. faecalis supernatants}

Following miRNA detection, we next sought to observe the protein expression alterations of BMSCs during this process. After performing proteomic analysis on BMSCs with or without $E$. faecalis supernatant treatment, we defined 63 significantly differentially expressed proteins by setting absolute level change $>2$ and $p$ value $<0.05$ as thresholds. All the differentially expressed proteins in BMSCs after E. faecalis supernatants treatment were listed in the heat map (Fig. 3a). Among these proteins,
38 proteins were upregulated and 25 proteins were downregulated. Next, gene ontology (GO) analysis showed that these proteins participated into a series of biological processes, such as the regulation of cell migration, the response to molecule of bacterial origin, and the positive regulation of cell motility (Fig. 3d). Furthermore, Kyoto Encyclopedia of Genes and Genomes (KEGG) pathway enrichment on the differentially expressed proteins were analyzed, and the top 20 enriched pathways, including NFkB signaling pathway, were shown (Fig. 3e).

miR-200a-3p affects the expressions of MMP-3 and MMP13 in BMSCs stimulated with $E$. faecalis supernatants As we chose to focus on the proteins involved in cell migration, further analyses on the mRNA levels of MMP-3 and MMP-13 were performed to identify their alterations in BMSCs with E. faecalis supernatant treatment. After cells were stimulated with the indicated concentration of E. faecalis supernatants for $24 \mathrm{~h}$, a concentrationdependent manner was obtained at mRNA expression level (Fig. 4a). Compared with the control group, the expressions of MMP-3 and MMP-13 showed a significant upregulation with increasing concentration of $E$. faecalis supernatants. Next, western blot results demonstrated that the expressions of MMP-3 and MMP-13 in BMSCs at protein level could also be promoted by E. faecalis supernatants (Fig. 4b and c). Further detection on the protein expressions of MMP-3 and MMP-13 showed that they were obviously upregulated with miR-200a-3p restoration, 

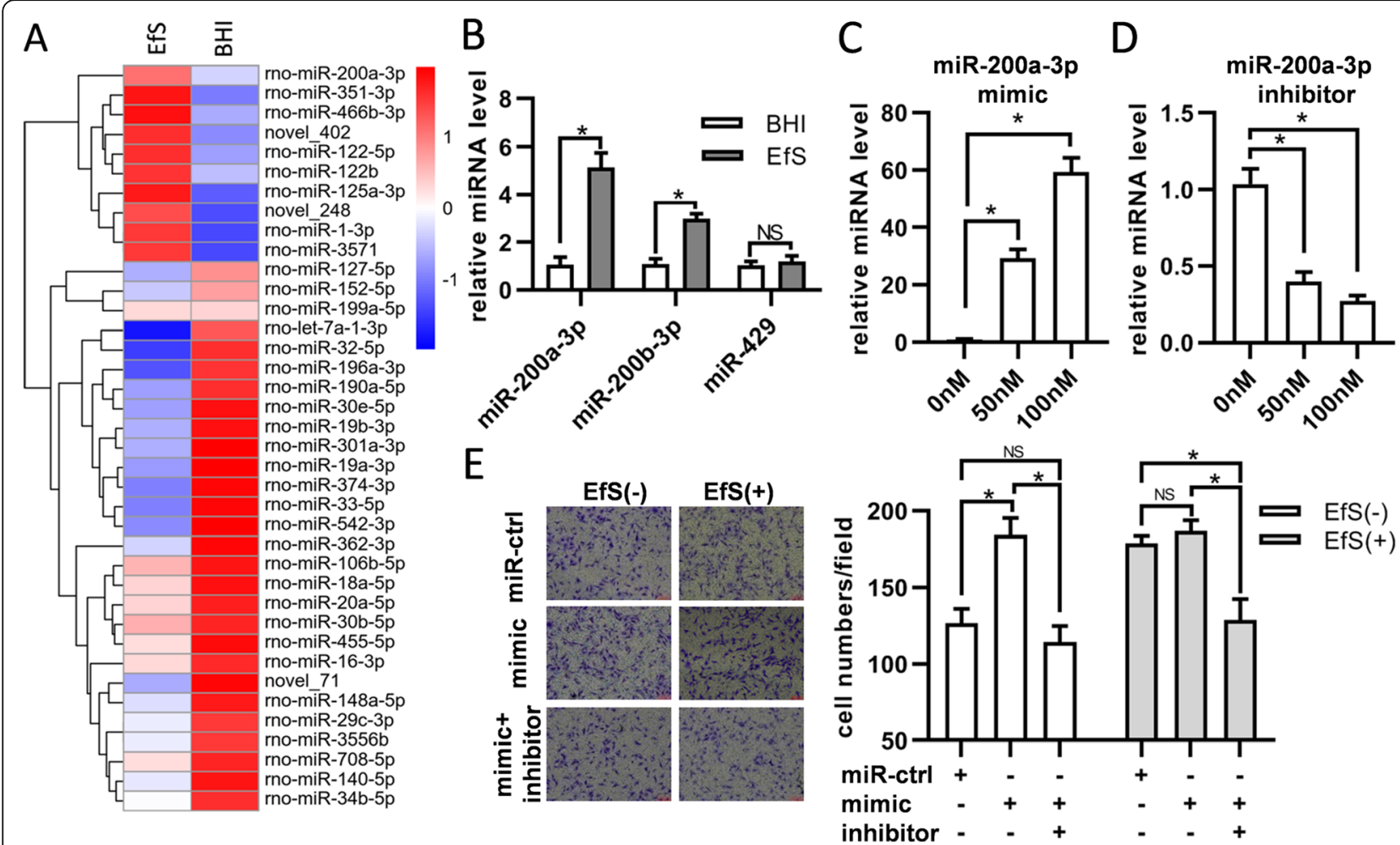

Fig. 2 miR-200a-3p was increased in Enterococcus faecalis supernatants stimulated BMSCs. a Heatmap of differential microRNA (miRNA) expression between BHI and EfS-treated BMSCs. Gene expression data were obtained with miRNA sequence and the most obvious values are shown. Red indicates increase, while blue means decrease. $\mathbf{b}$ Expression of miR-200 family members determined with qRT-PCR in EfS-treated BMSCs. c BMSCs were transfected with miR-200a-3p mimics with the concentration of $50 \mathrm{nM}$ and $100 \mathrm{nM}$. The expressions of miR-200a-3p were detected by qRT-PCR. $\mathbf{d}$ BMSCs were transfected with miR-200a-3p inhibitors with the concentration of $50 \mathrm{nM}$ and $100 \mathrm{nM}$. The expressions of miR-200a-3p were detected by qRT-PCR. In $\mathbf{b}$, $\mathbf{c}$, and $\mathbf{d}$, U6 RNA was taken as internal control. e Observation of BMSCs migration with the transfection of miR-200a-3p mimics and inhibitors. After transfection, cells were treated as in Fig. 1b and those cells migrated to the lower side of transwell membrane were counted after $12 \mathrm{~h} .{ }^{*} p<0.05$; NS, not significant

while inhibition of miR-200a-3p decreased their expressions (Fig. 4d, e).

\section{miR-200a-3p downregulates the expression of FOXJ1 by binding to its $3^{\prime} U T R$}

To further identify the functional target of miR-200a-3p, data were collected through public algorithms, and computational prediction reminded that miR-200a-3p downregulates FOXJ1 expression by directly binding to its 3 ' UTR (Fig. 5a). To verify the repression of FOXJ1 by miR-200a-3p binding to its 3'UTR, luciferase report assays containing either the wild-type or mutant FOXJ1 3' UTR sequence were conducted (Fig. 5b). The luciferase activities of wild-type FOXJ1 3'UTR reporter were repressed with overexpression of miR-200a-3p, when compared with the control groups (Fig. 5c). Furthermore, we found that BMSCs showed a dramatic decrease of FOXJ1 with E. faecalis supernatant treatment (Fig. 5d). No matter with or without $E$. faecalis supernatant treatment, miR-200a-3p mimics could repress the expression of FOXJ1 while inhibitors showed converse function.
miR-200a-3p increases BMSCs migration through FOXJ1/ NFKB pathway

Considering that FOXJ1 acts as a repressor of NFKB activation and the proteomic analysis reminded that the NFkB pathway was included in this process, we sought to investigate whether FOXJ1/NFKB axis plays a role during the migration of BMSCs. Firstly, we determined the level of $\mathrm{NF}_{\mathrm{K} B} \mathrm{~B}$ activation by luciferase report assays and found that the activation of NFKB was obviously upregulated by $E$. faecalis supernatants (Fig. 6a). Furthermore, transfection of miR-200a-3p could also improve the activity of NFKB (Fig. 6b). Following miR-200a-3p transfection, the expressions of $\mathrm{p}-\mathrm{NFKB}$ in BMSCs were detected by immunofluorescence. Compared with the control group, cells transfected with miR-200a-3p mimics showed more $\mathrm{p}-\mathrm{NF} \kappa \mathrm{B}$ expression in the cell nucleus (Fig. 6c). Consistent with the expressions of MMP3 and MMP-13, the expression of p-NFkB could also be increased by miR-200a-3p mimics (Fig. 6d). Meanwhile, transwell assays reminded that inhibition of $\mathrm{NFKB}_{\mathrm{B}}$ with PDTC would attenuate the migration of BMSCs (Fig. 


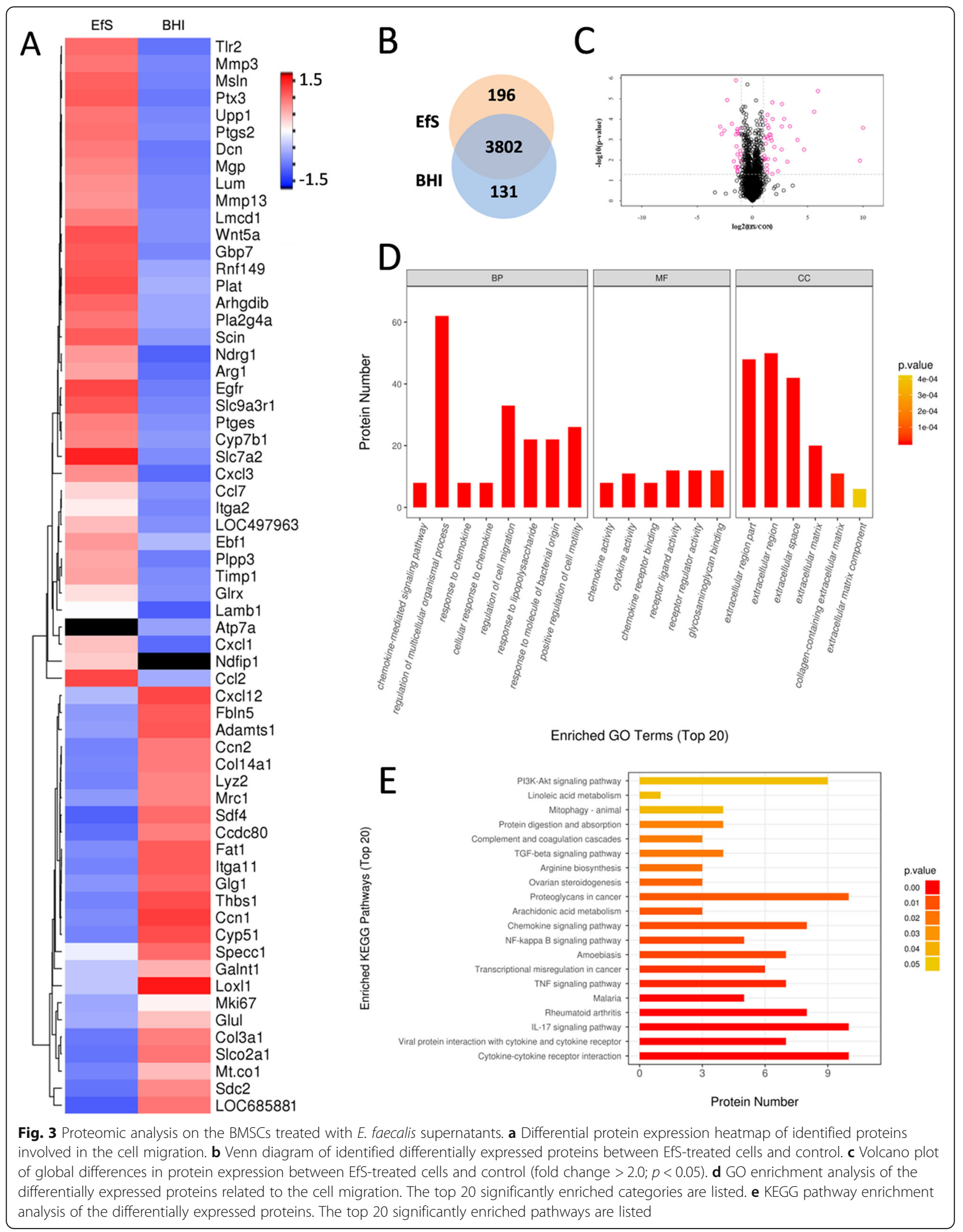




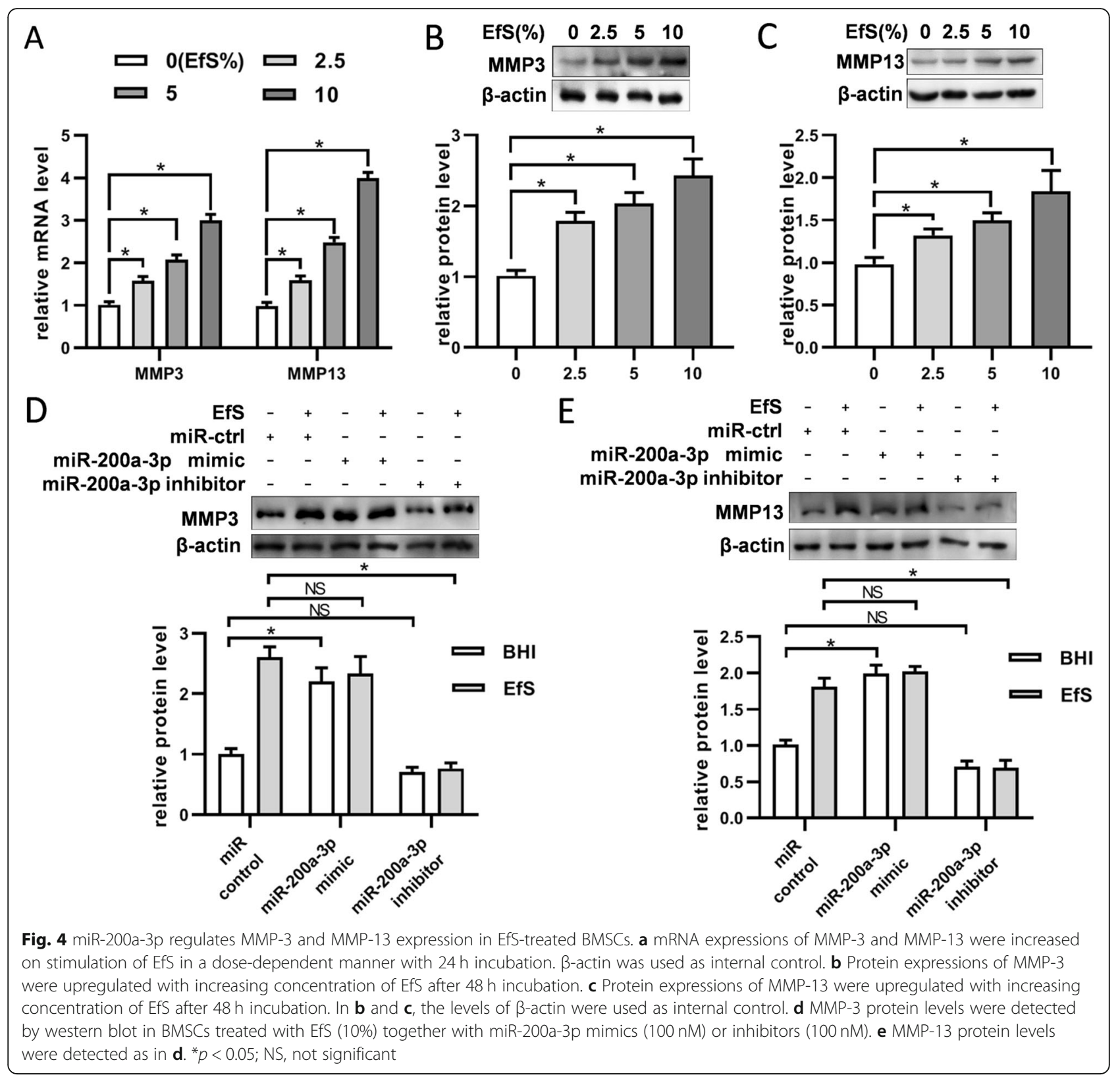

$6 e)$. And the upregulation of $\mathrm{p}-\mathrm{NF} \kappa \mathrm{B}, \mathrm{MMP}-3$, and MMP-13 induced by miR-200a-3p mimics could be diminished by PDTC (Fig. 6f-h).

\section{Discussion}

With multiple differentiation potential, BMSCs play an important role during the repair of bone lesion at the injured sites of apical periodontitis. The BMSCs reside in the stem cell niches at bone marrow and it is necessary for them to migrate to the damaged area and differentiate into osteoblasts to regenerate new tissues. Previous studies have described that BMSCs could be chemoattracted by the inflammation factors around the injured tissues $[25,26]$. However, it is still unclear whether the migration of BMSCs could be affected by the substances from microbes. Herein, we found that cell-free culture supernatants from the late stationary phase of $E$. faecalis could increase the migration of BMSCs when compared with the BHI medium. It reminds us that the secreted molecules or debris of E. faecalis could activate the motility of BMSCs.

A growing number of evidences have shown that miRNAs contribute to the migration of BMSCs under both physiological and pathological situation [22, 27]. To explore whether specific miRNAs were involved in this study, we detected the miRNA alterations of BMSCs treated with $E$. faecalis supernatants by miRNA sequencing. On the top of the list of upregulated miRNAs, 

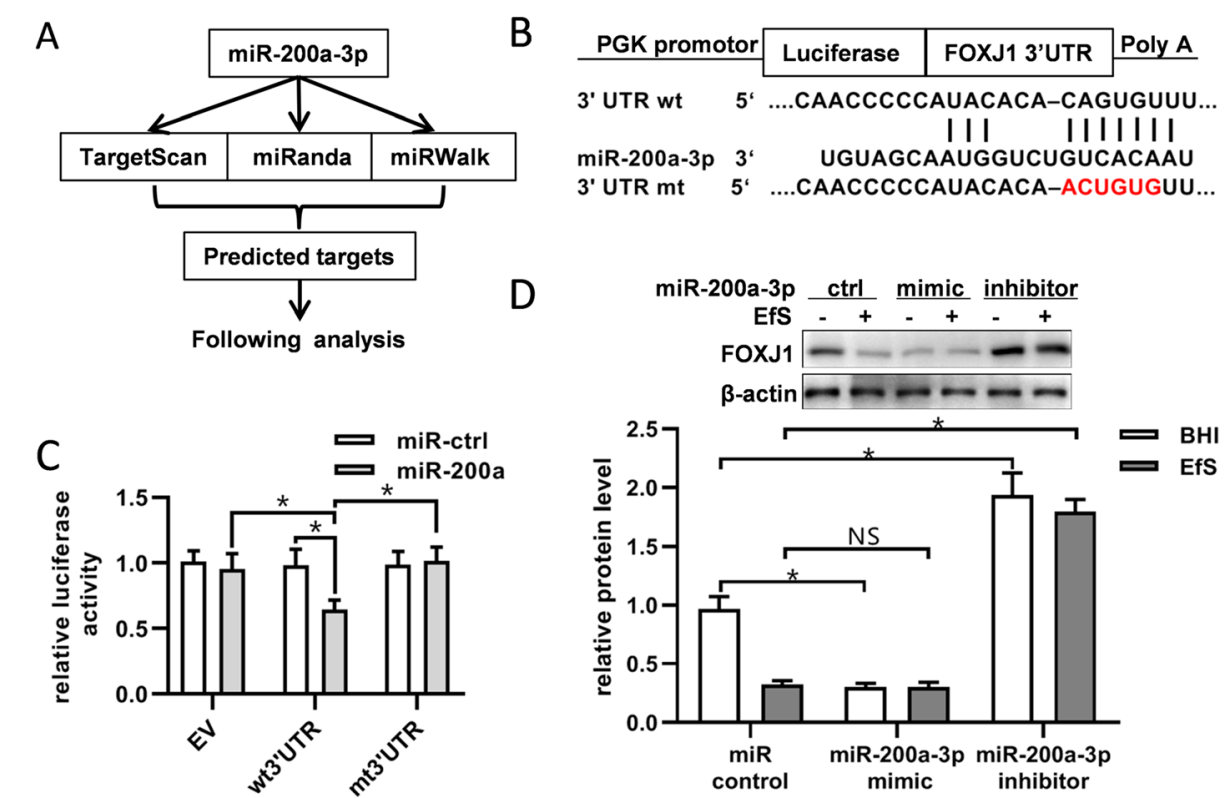

Fig. 5 miR-200a-3p decreases the expression of FOXJ1 by binding to its 3'-untranslated region. a Prediction on the potential targets of miR-200a$3 p$ using three miRNA target databases. b Wild type and mutation of the predicted miR-200a-3p binding sites at the $3^{\prime} U T R$ of FOXJ1. c The empty vector, wild-type or mutant reporter plasmids were transfected into HEK293T cells, together with miR-ctrl or miR-200a-3p. Luciferase reporter assays were used to identify the binding of miR-200a-3p to FOXJ1 3'UTR. d FOXJ1 protein levels were detected by western blot in BMSCs treated with or without EfS (10\%), together with miR-200a-3p mimics (100 nM) or inhibitors (100 nM). * $p<0.05$; NS, not significant

miR-200a-3p was noticed. Various studies have demonstrated the participation of miR-200 family in cell migration, and inhibition of miR-200 family members may downregulate the migration of cells [28]. In the present study, the migration of BMSCs was increased after miR200a-3p mimic transfection, while this promotion could be inhibited when the inhibitors of miR-200a-3p were applied. Together with the results above, these observations provided clues that miR-200a-3p takes part in the regulation of BMSCs migration caused by E. faecalis.

Considering that cell motility mainly relies on the protein rearrangements, we performed proteomic analysis to detect the molecular mechanisms underlying BMSCs migration and those proteins with statistical alterations were listed. Among the proteins which were highly expressed in the EfS treated groups, we found MMP-3 and MMP-13 were included. Matrix metalloproteinases (MMPs) belong to a large family and play critical roles in the tissue remodeling and extracellular matrix (ECM) degradation [29]. Various physiological and pathological processes, including cell migration and invasion, require the involvement of MMPs [30]. It has been revealed that MMP-3 and MMP-13 mediate the remodeling of ECM and contribute to the metastasis of cancer cells [31, 32]. Increased expression of MMP-3 and MMP-13 are associated with the augmentation of cell migration in lung cancer [33]. Previous studies have shown that the migration of colorectal cancer cells would be attenuated with downregulating MMP-3 [34], and knockdown either MMP-3 or MMP-13 could repress the invasion and migration of anaplastic thyroid carcinoma cells [35]. At the same time, knockdown of MMP-13 could dramatically downregulate the migration of ESCC (esophageal squamous cell carcinoma) cells [36]. An in vitro study on the adult neural stem/progenitor cells (aNPCs) found an increased expression of MMP-3 during the migration in response to chemokines [37]. Further gene ontology (GO) enrichment analysis on BMSCs also demonstrated that the top 8 affected biological process (BP) contained a response to molecule of bacterial origin, positive regulation of cell motility, and so on. All the results above suggest that molecules in E. faecalis supernatants promote the migration of BMSCs through regulating the expression of MMP-3 and MMP-13.

Given that the expression of MMPs showed obvious alteration during the migration of BMSCs induced by $E$. faecalis, we sought to determine the effect of miR-200a on the expression of MMPs. Initially, the expressions of MMP-3 and MMP-13 were confirmed at mRNA and protein level, and their expressions showed a concentrationdependent manner with stimulation of E. faecalis supernatants. These results are consisted of what we have obtained in the proteomic analysis. Previous researches have revealed that miR-200 could affect the expression of MMPs [38]. In this study, BMSCs transfected with miR200a-3p mimics showed similar results with those treated 


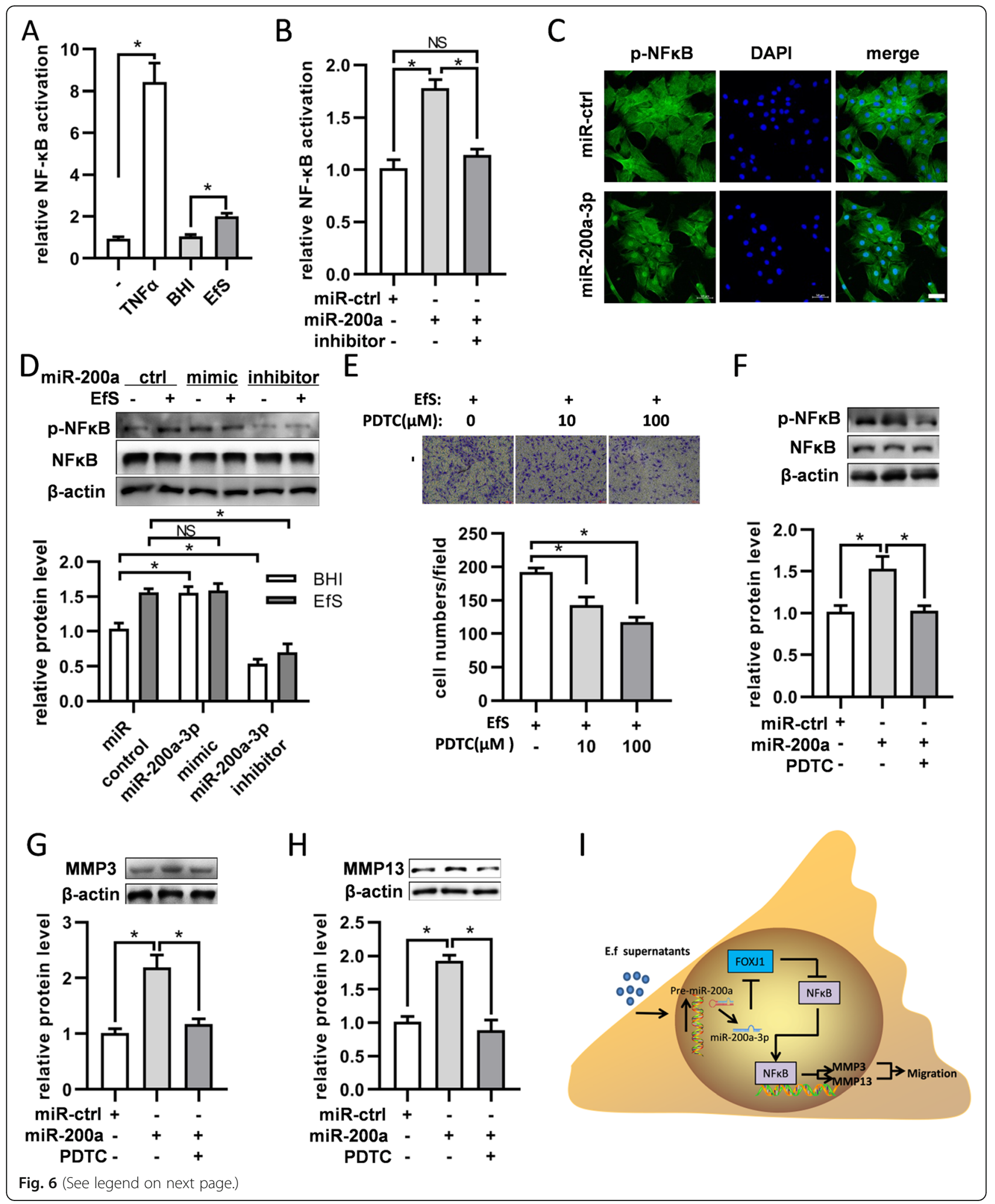


(See figure on previous page.)

Fig. 6 miR-200a-3p affects the expression of MMP-3/MMP-13 through regulating NFKB activity. a Increase of the NFkB activity by E. faecalis supernatants. The NFKB activation in the BMSCs treated with TNFa, BHI, or EfS were detected by luciferase assays with NFKB/uc reporter. The TNFa group was used as the positive control. $\mathbf{b}$ Promotion of the NFKB activity by miR-200a-3p transfection. Luciferase assays were used to detect the NFKB activation of BMSCs after transfection with miR-200a-3p mimics or inhibitors. $\mathbf{c}$ The expression of p-NFkB was increased in the nucleus of BMSCs with transfection of miR-200a-3p. $\mathbf{d}$ NFKB and p-NFKB levels were detected by western blot in BMSCs treated with EfS together with miR200a-3p mimics or inhibitors. Quantification of p-NFKB levels was calculated by normalizing with $\beta$-actin. e The migration of BMSCs could be attenuated by PDTC under the stimulation of EfS. DMSO was taken as the negative control. $\mathbf{f}$ Western blot analysis showed the effect of miR200a-3p on p-NFKB could be repressed by PDTC. Quantifications of p-NFKB level were calculated by normalizing with $\beta$-actin. $\mathbf{g}$ The expression level of MMP-3 increased by miR-200a-3p could be attenuated by PDTC. $\mathbf{h}$ The expression level of MMP-13 increased by miR-200a-3p could be attenuated by PDTC. In $\mathbf{g}$ and $\mathbf{h}$, quantification was calculated by normalizing with $\beta$-actin. i Schematic illustration implying the putative signaling pathways involved in the migration of BMSCs induced by E. faecalis supernatants. In response to $E$. faecalis supernatants, the transcription of miR$200 a-3 p$ is increased. Consequently, the expression of FOXJ1 is attenuated and its inhibition on nuclear factor kappa B (NFKB) pathway is abrogated. Activation and nucleus translocation of NFKB promotes the expressions of MMP-3 and MMP-13, which lead to the increased migration of BMSCs. ${ }^{*} p<0.05$; NS, not significant. In $\mathbf{c}$, bar $=50 \mu \mathrm{m}$

with E. faecalis supernatants in the expressions of MMP-3 and MMP-13. When miR-200a-3p inhibitors were applied, there was no obvious difference of MMP-3 and MMP-13 expression in comparison with the control group. However, the inhibitors could diminish the upregulation of MMP-3 and MMP-13 expression induced by mimics or EfS.

miRNA is usually functioned in a unique way, which means that a single miRNA can affect multiple RNA transcripts [39]. Thus, it is necessary to identify the target genes of miR-200a in BMSCs in order to detect the mechanism underlying BMSCs migration. Firstly, bioinformatics analysis was carried out to explore the potential targets of miR-200a-3p. Next, we identified FOXJ1 as one target gene of miR-200a by luciferases reporter assay. Previous studies have demonstrated that miR-200a could increase the migration of non-small cell lung cancer cells [40], while controversial results are also shown that the upregulation of miR-200a could suppress the migration of triplenegative breast cancer cells [41]. All the above reminds us that it may depend on the cell type how the miR-200a affects cell migration. FOXJ1, belonging to a DNA-binding protein family with the forkhead domain [42], could antagonize the $\mathrm{NF}_{\kappa} \mathrm{B}$ activation by inhibiting I $\mathrm{KB}$ protein [43]. Interestingly, we also observed the $N_{\kappa} B$ signaling pathway in the KEGG analysis.

The $\mathrm{NF}_{\kappa} \mathrm{B}$ transcription factor family forms various protein complexes and plays critical roles in the control of cell migration $[44,45]$. Previous studies have demonstrated that the NFKB/MMP-3 pathway plays roles in various cell migrations, including fibroblasts [46], prostate cancer cells [47], and chondrosarcoma cells [48], and so on. And NFKB/MMP-13 axis contributes to cell migration of lung cancer and glioma $[49,50]$. In our present study, both supernatants from E. faecalis and miR-200a-3p could promote the NFkB activation, while the inhibitor could suppress the activation. Consistent with the results that the MSC migration induced by IL1 $\beta$ could be impaired by the blockade of NFKB [51], we also found that inhibition of $\mathrm{NF}_{\mathrm{K}} \mathrm{B}$ activation could attenuate the migration of BMSCs, as well as the expressions of MMP-3 and MMP-13. Based on the findings above, we provided evidence that E. faecalis supernatants induce the BMSC migration through miR-200a-3p/ FOXJ1/NFkB/MMPs axis.

\section{Conclusions}

In this study, we provided evidence on the involvement of miR-200a-3p in the migration of BMSCs induced by E. faecalis supernatants and its downstream target FOXJ1. Furthermore, NFkB pathway activation was detected and contributed to the migration by promoting the expressions of MMP-3 and MMP-13 (see Fig. 6i for an overview). These findings provide a new perspective and may help understand the mechanism of BMSC migration in response to $E$. faecalis infection, though further investigations on other miRNAs involved in this process should be concerned.

\section{Abbreviations}

aNPCs: Adult neural stem/progenitor cells; BHI: Brain heart infusion; BMSCs: Bone marrow mesenchymal stem cells; DMEM: Dulbecco's modified Eagle's medium; EfS: Enterococcus faecalis supernatants; FBS: Fetal bovine serum; FOXJ1: Forkhead Box Protein J1; HRP: Horseradish peroxidase; LPS: Lipopolysaccharide; miRNA: Micro ribonucleic acid; MMP: Matrix metalloproteinases; NFkB: Nuclear factor kappa B; PBS: Phosphate buffer saline; PI3K: Phosphatidylinositol 3-kinase; PVDF: Polyvinylidene difluoride; TLR2: Toll-like receptor 2; UTR: Untranslated region

\section{Acknowledgements} Not applicable.

\section{Authors' contributions}

$M L$ and LW performed the majority of the experiments, designed the experiments, and wrote the manuscript. WZ participated in designing experiments and analyzed the data. ZH and SR assisted in conducting the experiments and discussing the results. $J$ conceived the project. All authors read and approved the final manuscript.

Authors' information

Not applicable. 


\section{Funding}

This work was supported by National Natural Science Foundation of China (No. 81271133).

\section{Availability of data and materials}

All miRNA sequence data supporting the conclusions of this article are available at [NCBI] repository under BioProject No. PRJNA602137. All mass spectrometry proteomics data are available in the [iProX partner] repository, [identifier PXD017438 and the hyperlink to data in http://proteomecentral. proteomexchange.org].

\section{Ethics approval and consent to participate}

All the procedures mentioned in this study were approved by the Institutional Ethical Review Board of Shanghai Ninth People's Hospital, School of Medicine, Shanghai Jiao Tong University, and conducted in conformity with institutional guidelines for the care and use of laboratory animals in School of Medicine, Shanghai Jiao Tong University.

\section{Consent for publication}

Not applicable.

\section{Competing interests}

The authors declare that they have no competing interests.

\section{Author details}

${ }^{1}$ Department of Endodontics and Operative Dentistry, Shanghai Ninth People's Hospital, College of Stomatology, Shanghai Jiao Tong University School of Medicine, Shanghai, China. ${ }^{2}$ National Clinical Research Center for Oral Diseases, Shanghai, China. ${ }^{3}$ Shanghai Key Laboratory of Stomatology \& Shanghai Research Institute of Stomatology, Shanghai, China.

\section{Received: 30 April 2020 Revised: 29 June 2020}

\section{Accepted: 14 July 2020 Published online: 25 July 2020}

\section{References}

1. Moller AJ, Fabricius L, Dahlen G, Ohman AE, Heyden G. Influence on periapical tissues of indigenous oral bacteria and necrotic pulp tissue in monkeys. Scand J Dent Res. 1981;89:475-84.

2. Sakko M, Tjaderhane $L$, Rautemaa-Richardson R. Microbiology of root canal infections. Prim Dent J. 2016;5:84-9.

3. Stuart CH, Schwartz SA, Beeson TJ, Owatz CB. Enterococcus faecalis: its role in root canal treatment failure and current concepts in retreatment. J Endod. 2006:32:93-8.

4. Chavez DPL, Davies JR, Bergenholtz G, Svensater G. Strains of Enterococcus faecalis differ in their ability to coexist in biofilms with other root canal bacteria. Int Endod J. 2015;48:916-25.

5. Peciuliene V, Reynaud AH, Balciuniene I, Haapasalo M. Isolation of yeasts and enteric bacteria in root-filled teeth with chronic apical periodontitis. Int Endod J. 2001:34:429-34.

6. Kayaoglu G, Orstavik D. Virulence factors of Enterococcus faecalis: relationship to endodontic disease. Crit Rev Oral Biol Med. 2004;15:308-20.

7. Fisher K, Phillips C. The ecology, epidemiology and virulence of Enterococcus. Microbiology. 2009;155:1749-57.

8. Jhajharia K, Parolia A, Shetty KV, Mehta LK. Biofilm in endodontics: a review. J Int Soc Prev Community Dent. 2015;5:1-12.

9. Hienz SA, Paliwal S, Ivanovski S. Mechanisms of bone resorption in periodontitis. J Immunol Res. 2015;2015:615486.

10. Raeth S, Sacchetti B, Siegel G, Mau-Holzmann UA, Hansmann J, Vacun G, et al. A mouse bone marrow stromal cell line with skeletal stem cell characteristics to study osteogenesis in vitro and in vivo. Stem Cells Dev. 2014;23:1097-108

11. Sacchetti B, Funari A, Michienzi S, Di Cesare S, Piersanti S, Saggio I, et al. Self-renewing osteoprogenitors in bone marrow sinusoids can organize a hematopoietic microenvironment. Cell. 2007;131:324-36.

12. Ito $\mathrm{H}$. Chemokines in mesenchymal stem cell therapy for bone repair: a novel concept of recruiting mesenchymal stem cells and the possible cell sources. Mod Rheumatol. 2011:21:113-21.

13. Tomchuck SL, Zwezdaryk KJ, Coffelt SB, Waterman RS, Danka ES, Scandurro $A B$. Toll-like receptors on human mesenchymal stem cells drive their migration and immunomodulating responses. Stem Cells. 2008;26:99-107.
14. Lei J, Wang Z, Hui D, Yu W, Zhou D, Xia W, et al. Ligation of TLR2 and TLR4 on murine bone marrow-derived mesenchymal stem cells triggers differential effects on their immunosuppressive activity. Cell Immunol. 2011; 271:147-56.

15. Liu Y, Gao Y, Zhan X, Cui L, Xu S, Ma D, et al. TLR4 activation by lipopolysaccharide and Streptococcus mutans induces differential regulation of proliferation and migration in human dental pulp stem cells. J Endod. 2014;40:1375-81.

16. Weber JA, Baxter DH, Zhang S, Huang DY, Huang KH, Lee MJ, et al. The microRNA spectrum in 12 body fluids. Clin Chem. 2010;56:1733-41.

17. Ivey KN, Srivastava D. microRNAs as developmental regulators. Cold Spring Harb Perspect Biol. 2015;7:a8144.

18. Macfarlane LA, Murphy PR. MicroRNA: biogenesis, function and role in cancer. Curr Genomics. 2010;11:537-61.

19. Jiang $Q$, Wang $Y$, Hao $Y$, Juan $L$, Teng $M$, Zhang $X$, et al. miR2Disease: a manually curated database for microRNA deregulation in human disease. Nucleic Acids Res. 2009:37:D98-104.

20. Kang $H$, Hata A. The role of microRNAs in cell fate determination of mesenchymal stem cells: balancing adipogenesis and osteogenesis. BMB Rep. 2015;48:319-23.

21. van Rooij E, Kauppinen S. Development of microRNA therapeutics is coming of age. Embo Mol Med. 2014;6:851-64.

22. Lv C, Yang $S$, Chen $X$, Zhu X, Lin W, Wang L, et al. MicroRNA-21 promotes bone mesenchymal stem cells migration in vitro by activating PI3K/Akt/ MMPs pathway. J Clin Neurosci. 2017;46:156-62.

23. He L, Wang X, Kang N, Xu J, Dai N, Xu X, et al. MiR-375 inhibits the hepatocyte growth factor-elicited migration of mesenchymal stem cells by downregulating Akt signaling. Cell Tissue Res. 2018;372:99-114.

24. Vasaikar S, Huang C, Wang X, Petyuk VA, Savage SR, Wen B, et al. Proteogenomic analysis of human colon cancer reveals new therapeutic opportunities. Cell. 2019;177:1035-49.

25. Park MS, Kim YH, Jung Y, Kim SH, Park JC, Yoon DS, et al. In situ recruitment of human bone marrow-derived mesenchymal stem cells using chemokines for articular cartilage regeneration. Cell Transplant. 2015;24:1067-83.

26. Auletta JJ, Deans RJ, Bartholomew AM. Emerging roles for multipotent, bone marrow-derived stromal cells in host defense. Blood. 2012;119:1801-9.

27. Bing $W$, Pang $X$, Qu Q, Bai X, Yang W, Bi Y, et al. Simvastatin improves the homing of BMSCs via the PI3K/AKT/miR-9 pathway. J Cell Mol Med. 2016;20:949-61.

28. Senfter D, Madlener S, Krupitza G, Mader RM. The microRNA-200 family: still much to discover. Biomol Concepts. 2016;7:311-9.

29. Di Girolamo N, Chui J, Coroneo MT, Wakefield D. Pathogenesis of pterygia: role of cytokines, growth factors, and matrix metalloproteinases. Prog Retin Eye Res. 2004;23:195-228.

30. Kapoor C, Vaidya S, Wadhwan V, Kaur G, Pathak A. Seesaw of matrix metalloproteinases (MMPs). J Cancer Res Ther. 2016;12:28-35.

31. Slattery ML, John E, Torres-Mejia G, Stern M, Lundgreen A, Hines L, et al. Matrix metalloproteinase genes are associated with breast cancer risk and survival: the Breast Cancer Health Disparities Study. PLoS One. 2013;8:e63165.

32. Balbin $M$, Pendas AM, Uria JA, Jimenez MG, Freije JP, Lopez-Otin C. Expression and regulation of collagenase-3 (MMP-13) in human malignant tumors. Apmis. 1999;107:45-53.

33. Jiang YN, Yan HQ, Huang XB, Wang YN, Li Q, Gao FG. Interleukin 6 trigged ataxia-telangiectasia mutated activation facilitates lung cancer metastasis via MMP-3/MMP-13 up-regulation. Oncotarget. 2015;6:40719-33.

34. Bufu T, Di X, Yilin Z, Gege L, Xi C, Ling W. Celastrol inhibits colorectal cancer cell proliferation and migration through suppression of MMP3 and MMP7 by the PI3KJAKT signaling pathway. Anti-Cancer Drugs. 2018;29:530-8.

35. Ma Y, Cang S, Li G, Su Y, Zhang H, Wang L, et al. Integrated analysis of transcriptome data revealed MMP3 and MMP13 as critical genes in anaplastic thyroid cancer progression. J Cell Physiol. 2019;234:22260-71.

36. Osako Y, Seki N, Kita Y, Yonemori K, Koshizuka K, Kurozumi A, et al. Regulation of MMP13 by antitumor microRNA-375 markedly inhibits cancer cell migration and invasion in esophageal squamous cell carcinoma. Int J Oncol. 2016:49:2255-64

37. Barkho BZ, Munoz AE, Li X, Li L, Cunningham LA, Zhao X. Endogenous matrix metalloproteinase (MMP)-3 and MMP-9 promote the differentiation and migration of adult neural progenitor cells in response to chemokines. Stem Cells. 2008:26:3139-49.

38. Bracken CP, Li X, Wright JA, Lawrence DM, Pillman KA, Salmanidis M, et al. Genome-wide identification of miR-200 targets reveals a regulatory network controlling cell invasion. EMBO J. 2014;33:2040-56. 
39. Kinoshita T, Yip KW, Spence T, Liu FF. MicroRNAs in extracellular vesicles: potential cancer biomarkers. J Hum Genet. 2017;62:67-74.

40. Chen Y, Peng W, Lu Y, Chen J, Zhu YY, Xi T. MiR-200a enhances the migrations of A549 and SK-MES-1 cells by regulating the expression of TSPA N1. J Biosci. 2013;38:523-32.

41. Tsouko E, Wang J, Frigo DE, Aydogdu E, Williams C. miR-200a inhibits migration of triple-negative breast cancer cells through direct repression of the EPHA2 oncogene. Carcinogenesis. 2015;36:1051-60.

42. Lam EW, Brosens JJ, Gomes AR, Koo CY. Forkhead box proteins: tuning forks for transcriptional harmony. Nat Rev Cancer. 2013;13:482-95.

43. Lin L, Spoor MS, Gerth AJ, Brody SL, Peng SL. Modulation of Th1 activation and inflammation by the NF-kappaB repressor Foxj1. Science. 2004;303: 1017-20.

44. Oeckinghaus A, Ghosh S. The NF-kappaB family of transcription factors and its regulation. Cold Spring Harb Perspect Biol. 2009:1:a34.

45. Li Q, Verma IM. NF-kappaB regulation in the immune system. Nat Rev Immunol. 2002;2:725-34.

46. Authier H, Billot K, Derudder E, Bordereaux D, Riviere P, Rodrigues-Ferreira $\mathrm{S}$, et al. IKK phosphorylates RelB to modulate its promoter specificity and promote fibroblast migration downstream of TNF receptors. Proc Natl Acad Sci U S A. 2014;111:14794-9.

47. Qu H, Zou Z, Pan Z, Zhang T, Deng N, Chen G, et al. IL-7/IL-7 receptor axis stimulates prostate cancer cell invasion and migration via AKT/NF-kappaB pathway. Int Immunopharmacol. 2016;40:203-10.

48. Tang CH, Yamamoto A, Lin YT, Fong YC, Tan TW. Involvement of matrix metalloproteinase-3 in CCL5/CCR5 pathway of chondrosarcomas metastasis. Biochem Pharmacol. 2010;79:209-17.

49. Liu JF, Lee CW, Tsai MH, Tang CH, Chen PC, Lin LW, et al. Thrombospondin 2 promotes tumor metastasis by inducing matrix metalloproteinase-13 production in lung cancer cells. Biochem Pharmacol. 2018;155:537-46.

50. Yeh WL, Lu DY, Lee MJ, Fu WM. Leptin induces migration and invasion of glioma cells through MMP-13 production. Glia. 2009;57:454-64.

51. Carrero R, Cerrada I, Lledo E, Dopazo J, Garcia-Garcia F, Rubio MP, et al. IL 1 beta induces mesenchymal stem cells migration and leucocyte chemotaxis through NF-kappaB. Stem Cell Rev Rep. 2012;8:905-16.

\section{Publisher's Note}

Springer Nature remains neutral with regard to jurisdictional claims in published maps and institutional affiliations.

Ready to submit your research? Choose BMC and benefit from:

- fast, convenient online submission

- thorough peer review by experienced researchers in your field

- rapid publication on acceptance

- support for research data, including large and complex data types

- gold Open Access which fosters wider collaboration and increased citations

- maximum visibility for your research: over $100 \mathrm{M}$ website views per year

At $\mathrm{BMC}$, research is always in progress.

Learn more biomedcentral.com/submissions 